ISAHP 1996, Vancouver, Canada, July 12-15, 1996

\title{
AN APPLICATION OF AHP METHOD TO MARKOV DECISION PROCESS
}

\author{
Liang Zhaoning, Li Changmin and Qu Yannian \\ Northeast University, Shenyang 110015, China
}

\section{INTRODUCTION}

It is very important to carry out macroscopic control for industry economy in the course of the operation of the market mechanism. This paper iritroduces the integrated model of AHP method and Markov decision process so as to provide the accordance and information on quantitative analysis to make an economic development plan.

\section{PROBLEMS}

In case, a decision department has some subordinate enterprise groups, it puts respectively two kinds of management policies to the subordinate enterprises. The first, it transfers its power to the enterprises that accept the market regulation; the other. it imiplements the insturtivs policy of macroscopic control and manages all the things done by its subordinates. These two kinds of management policies are assinied as $U_{1}$ and $U_{2}$ that represent respectively the two parts of ratio relationship. If $U_{1}+U_{2}=1, U_{1}, U_{2} \geqslant 0$, the problem is what ratio $U_{1}$ and $U_{2}$ should respectively possess in the total industry.

\section{ESTABLISHAENT OF MATHEMATICAL MODEL}

There are more than three thousand subordinate' enterprises in Shenyang city, Liaoning Province, China. If all of them operate entirely according to tie market regulation, many enterprises will have noi any adaptive ability and go into bankruptcy; if all of them operate entirely according to the planned economy, the government in Shenyang has no capability unough. For this reason we apply the above-mentioned two kinds of poifies $\mathrm{U}_{1}, \mathrm{U}_{2}$. We have analyzed the state of these entrprises, and we divide them into five circumstances according to our investigation:

$S_{1}$ : developing rapidly: $S_{2}$ : developing proportionally; $S_{3}$ : remaining the status quo; $S_{4}$ : shrinking $30 \%: S_{5}$ : shrinking more than $70 \%$. 
We establish the network structure model as follows:

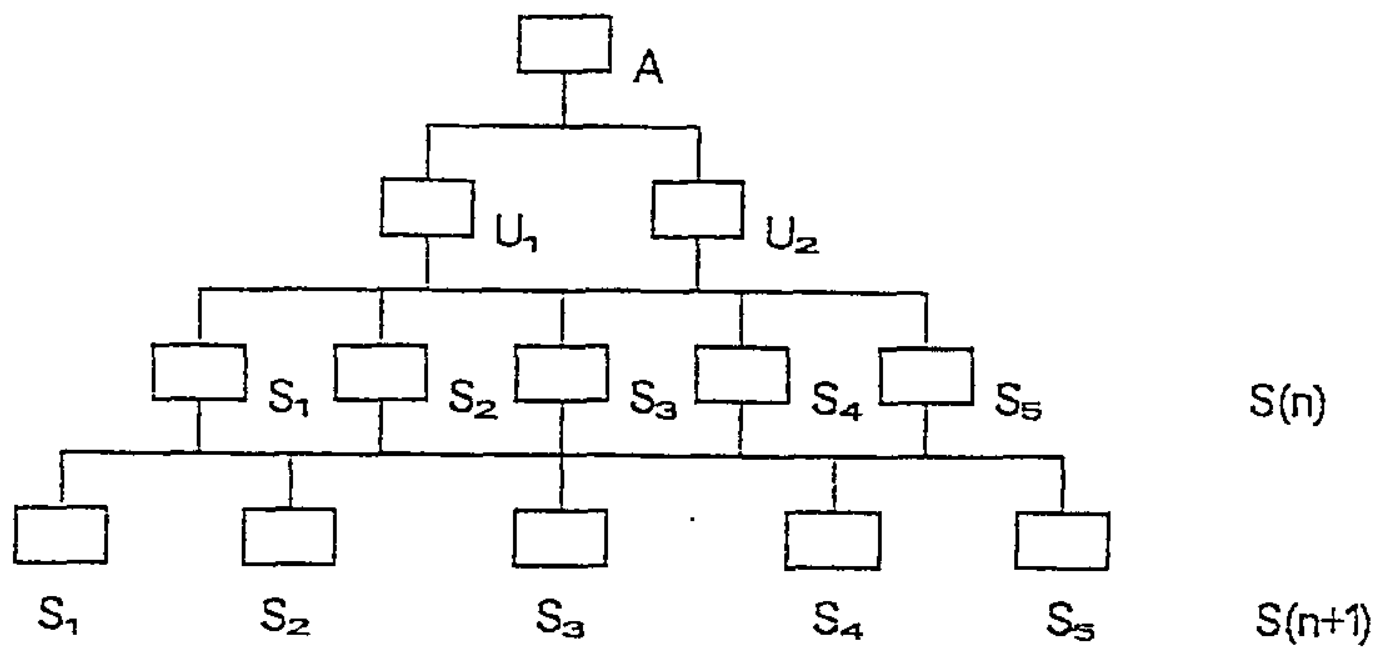

Figure 1 Network Structure

Assuming that $U=\left(U_{1}, U_{2}\right)^{\top}$, then. $S(n)=\left\{S_{1}(n) . S_{2}(n) \ldots, S_{n}(n+1)\right\} T$, and $S(n+1)=\left\{S_{1}(n+1), S_{2}(n+1), \ldots, S_{n}(n+1)\right\} T$.

In this formula, $S=\left(S_{1}, \ldots, S_{m}\right)$ is called a state vector, $n$ is time which is measured by the year, $m=5, S_{1} \ldots ., S_{5}$ indicates five sorts of states meritioned above.

The transfer probability matrix from state $S(n) \cdot$ tc $S(n+1)$ is $\left\{P_{i j}(n)\right\}, i=1,2 \ldots, 5 ; j=1,2, \ldots, 5$.

The probability distribution of state $S(n)$ in $U_{1}$ is $\beta_{11}(n), \beta_{12}(n) \ldots$, $\beta_{15}(n)$.

The probability distribution of state $S(n)$ in $U_{2}$ is $\beta_{21}(n), \beta_{22}(n) \ldots$, $\beta_{25}(n)$.

$U_{1}$ and $U_{2}$ are the policies adopted by the decision department $A$. Through Markov process, it is known:

$W(n+i)=P(n) B(n)\left(U_{1}, U_{2}\right) T$. In this formula. $W(n+1)$ is the probability distribution of $S(n+1)$.

It is assumed that the profit vector of $S(n)=\left[S_{1}(n), S_{2}(n), \ldots, S_{5}(n)\right]$ is $C(n)=\left[Q_{1}(n), Q_{2}(n), \ldots, Q_{5}(n)\right]$; then, the total profit earch year is

$$
C(n)=\sum_{i=1}^{5} W_{i}(n) Q_{i}(n)
$$

3. THE ESTIHATED PARARETER FOR AHP -.- B(N). P(N) In object $U_{1}$, the reletive veighted value of $S_{1}, \ldots, S_{5}$ is $\beta_{11}(n), \ldots$, 
$\beta_{15}(n)$ : in object $U_{2}$, the relative weighted value of $S_{1}, S_{2}, \ldots, S_{5}$ is $\beta_{27}(n), \ldots . \beta_{25}(n)$. Similarly. the relative weighted value of $S(n+1)$ in $S(n)$ can be estimated with AHP method, and the matrix $\left\{P_{i j}(n)\right\} . i . j=1.2 \ldots . . m$. is obtained.

It is assumed that $U_{2}=X$. as a result, $U_{1}=1-X$, here. $0 \leqslant X \leqslant 1$ : then.

$W(n+1)=P(n) \beta(n)\left(U_{1}, U_{2}\right)^{\top}$ can also be known in $U+1$ years. The total profit of each year is

$$
C(n)=\sum_{i=1}^{5} W_{i}(n) Q_{i}(n)
$$

The parameter in the Markov decision process can completely be estimated with AHP method. therefore. the analysis for Markov decision process can be carried out.

\section{DECISION ANALYSIS ALGORITHM}

Markov decision process is shown in the following figure:

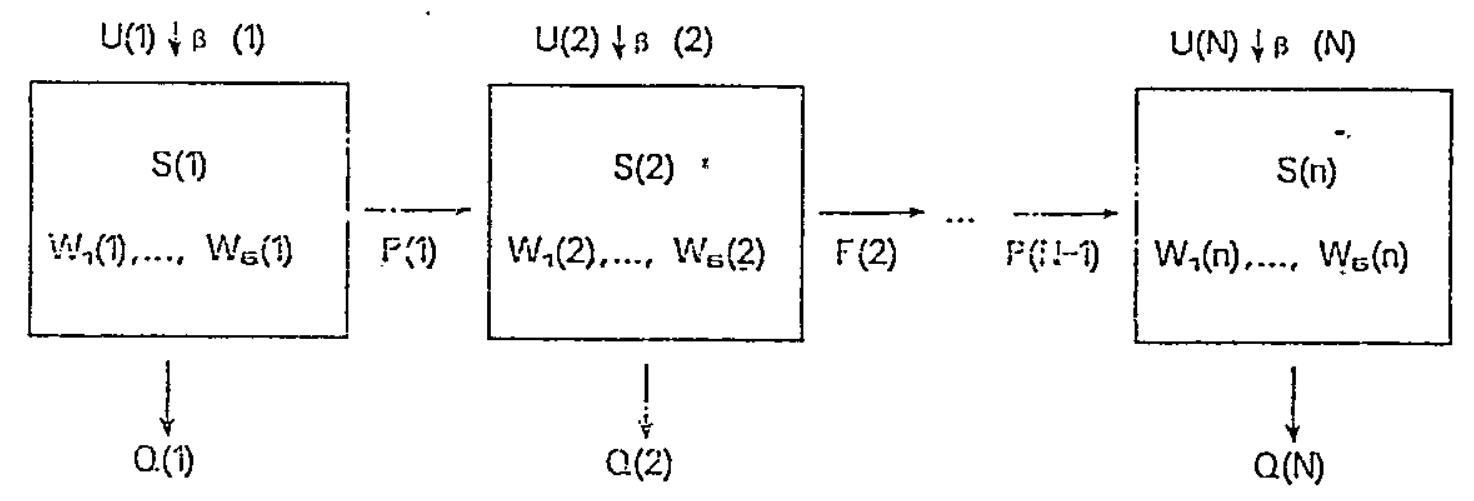

Figure 2 Markov Deçision Process

In Figure 2, $n=1$ is an initial state, which indicates one year; when $n$ is equal to 2, the relative weighted value of $S(2)$ in $U(2)$ is $\beta(2)$. Through the transfer matrix of $P(1)$ from $S(1)$ to $S(2), W(2)=P(1) \beta(1)\left(U_{1}, U_{2}\right)^{\top}$ can be calculated. After that, base on the calculated $\beta(2), P(2)$ and the given $U_{1}, U_{2} . W(3)$ can also be caiculated. Similarly, the expression $W(4)$. $W(5)$ can be calculated in turn. Because $U_{2}(n)=X_{n}, U_{1}(n)=1-X_{n}$ is assumed each time, the state equation for making an $n$ year plan is :

$W(n+1)=P(n) \beta(n)\left(U_{1}(n), U_{2}(n)\right)^{\top}$;

Object function:

$J_{V V} \cdots \sum_{n=1}^{5} C(n), \quad C(n)=\sum_{i=1}^{5} W_{i}(n) Q_{i}(n)$

According to Bellman dynamic programming. 


$$
J^{*} n-j=\underset{0<x \leqslant 1}{\operatorname{Max}\{C n-j+J n-j+1 *\} . j=0,1,2 \ldots, i i}
$$

So, $U_{2}(n)=X_{n}, U_{1}(n)=1-X_{n}$ can be solved as the basis for decision department to make a policy.

\section{CONCLUSION}

In accordance with the investigation and calculation in three thousand enterprises in Shenyang, Liaoning Province. China.

$W(n), Q(n) . n=1,2,3,4.5$, is obtained.

The optimal solution of $\left\{U_{2}(n)=\times_{n}\right\}\left\{U_{i}(n)=1-\times_{n}\right\}$ have been obtained with Bellman algorithm in following figure:

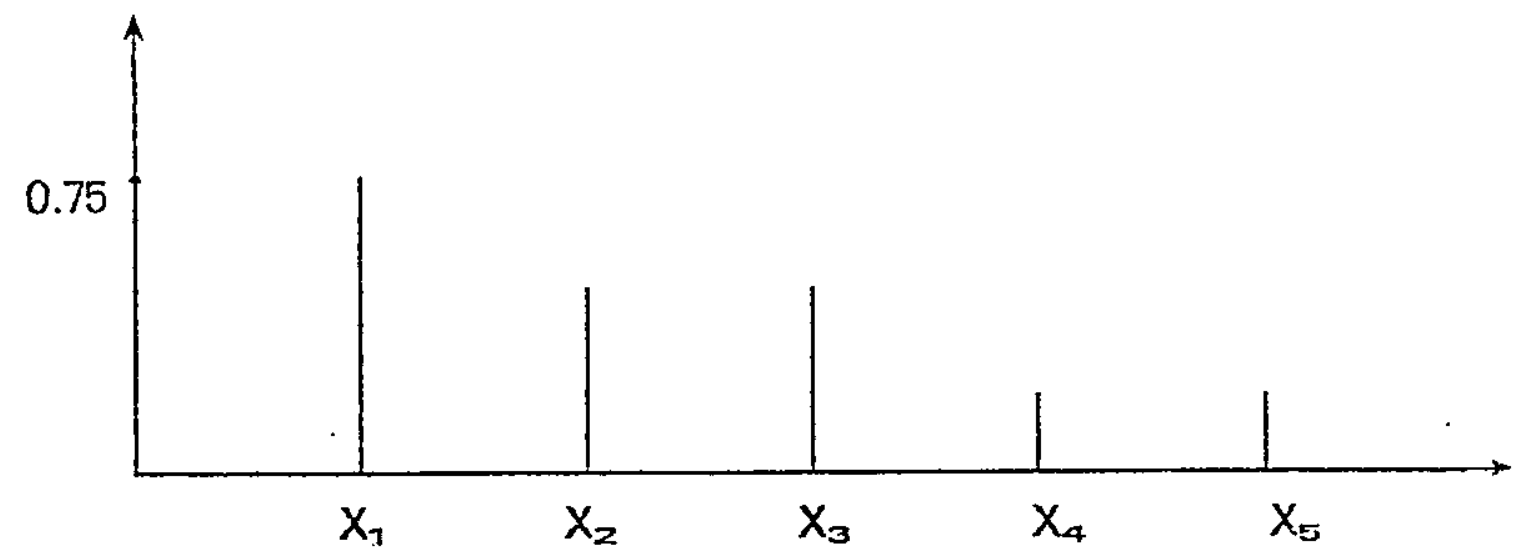

Figure 3

\section{REFERENCES}

1 Xu Shubo. The Analytic Hierarchy Process. Tianjin University Press. 1988

2 Saaty T.L. The Analytic Hierarchy Process. McGraw Hill. 1980 